\title{
A bead-based microfluidic system for joint detection in TORCH screening at point-of-care testing
}

\author{
Xianbo Qiu ${ }^{1}$ Junhui Zhang ${ }^{1}$ Yichen $\mathrm{Li}^{1}$ - Chaomin Zhang ${ }^{1}$ - Dong Wang ${ }^{2}$. \\ Weiling $\mathrm{Zhu}^{2} \cdot \mathrm{Fang}^{2}{ }^{2} \cdot$ Shengxiang $\mathrm{Ge}^{3} \cdot \mathrm{Ningshao} \mathrm{Xia}^{3} \cdot$ Shizhi Qian $^{4}$
}

Received: 10 October 2017 / Accepted: 14 October 2017/Published online: 20 October 2017

(C) Springer-Verlag GmbH Germany 2017

\begin{abstract}
A concise bead-based microfluidic system has been developed for joint detection in TORCH screening at point-of-care testing. Assisted by five functionalized polycarbonate single beads, totally five critical infections including TOX, RUB, CMV, HSV-1 and HSV-2 can be simultaneously detected with the microfluidic chip based on chemiluminescence. The microfluidic chip consists of multiple reaction chambers respectively for different reaction steps of the sandwiched chemiluminescence immunoassay, and each reaction chamber is divided into five interconnected independent cells to respectively hold five single beads in different position. With a guiding path, each single bead with a ferrous core can be easily transported between each two adjacent reaction chambers through magnetic control to consecutively react with the serum sample or other different reagents. Meanwhile, with magnetic control, each single bead within the reaction chamber can be moved back and forth to improve the detection sensitivity with enhanced mixing. In one time test, joint detection with five infections to one serum sample can be simultaneously implemented by introducing
\end{abstract}

Xianbo Qiu

xbqiu@mail.buct.edu.cn

1 Institute of Microfluidic Chip Development in Biomedical Engineering, College of Information Science and Technology, Beijing University of Chemical Technology, Beijing 100029, China

2 Beijing Wantai Biological Pharmacy Enterprise Co., Ltd., Beijing 102206, China

3 National Institute of Diagnostics and Vaccine Development in Infectious Diseases, Xiamen University, Xiamen 361005, China

4 Institute of Micro/Nanotechnology, Old Dominion University, Norfolk, VA 23529, USA synchronous manipulation to five single beads. Chemiluminescence signal from five single beads is collected by a sensitive CCD camera in parallel. Qualitative detection is achieved by comparing the detection signal amplitude with the corresponding cutoff value for each infection. Experimental results show that accurate, simple, convenient and efficient TORCH screening can be achieved with the easyto-operate and low-cost microfluidic system at resourcepoor settings.

\section{Introduction}

As a promising technology to automate, miniaturize and integrate the bio-analytical processes, 'lab-on-a-chip' or microfluidic systems have been intensively studied to develop a new diagnosis platform with efficient, convenient, rapid, easy-to-operate and low-cost detection systems (Stumpf et al. 2015; Sun et al. 2014; Mosley et al. 2016). With the integration of micro-pump, micro-valve, micro-channel, micro-reactor and other functional modules, a microfluidic system is able to allow multiple reaction steps for a specific bio-analysis to be consecutively performed without or with less human interference (Chen et al. 2010; Choi et al. 2016; Qiu et al. 2011a, b). For example, with different integrated microfluidic systems, immunoassay based HIV detection can be performed with an automatic timer actuator or just by simple finger actuation (Liu et al. 2009; Qiu et al. 2009). With fully integrated microfluidic systems, for example, 'sample-in and answer-out' nucleic acid diagnosis chips, even nucleic acid based analysis with much complicated procedure can be performed in a simple, convenient, easy-to-operate and low-cost way (Qiu et al. 2011a, b; Du et al. 2017; Zhang et al. 2017). As another typical representative, paper-based 
microfluidic systems are able to implement bio-analysis in an even more convenient way without active pumping, which is helpful to get rid of the complicated and bulky actuation device (Wu et al. 2017; López-Marzo and Merkoçi 2016; Gong and Sinton 2017). Furthermore, with smartphone based detection, simple and convenient diagnosis with disposable microfluidic chips can be performed at resource poor settings or even at home at point-of-care testing (Li et al. 2016; Zhang and Liu 2016; Yang et al. 2016).

Various outcomes, for example, fetal death, organ injury, or limited sequelae can be caused when pathogen related infections are acquired in utero or in the immediate post-natal period with pregnant women (Neu et al. 2015; Barbi et al. 2006). Typically, the TORCH is referred as a classical group of five teratogenic pathogens including Toxoplasmosis (TOX), Rubella virus (RUB), Cytomegalovirus (CMV), and Herpes simplex virus type 1 and 2 (HSV-1, HSV-2) (Jong et al. 2013). TORCH screening to the pregnant women is helpful to provide critical clinical information for antenatal care (Li et al. 2006). Different methods, for example, ELISA (enzyme linked immunosorbent assay), CLIA (chemiluminescence), ECLIA (electrochemiluminiscence) based on IgG or IgM have been developed for TORCH screening (Ursu et al. 2015). Automated instruments are developed to implement screening for RUB or CMV infection in pregnancy to achieve convenient, rapid and reliable diagnosis with significantly reduced detection time (Van et al. 2014; Revello et al. 2012). A rapid and low-cost TORCH screening method based on microarrays possessing comparable sensitivity and specificity with ELISA has been developed (Yang et al. 2009). Similarly, another method based on microarrays with fluorescent or colorimetric labeling for serodiagnosis of TORCH infections has been developed (Jiang et al. 2008).

Bead-based immunoassay on microfluidic chip is regarded as a unique platform for high performance diagnosis based on its distinct characteristics of great flexibility, a high surface-to-volume ratio, and remarkable compatibility with high throughput analysis (Lim and Zhang 2007; Chung et al. 2016; Zhang et al. 2013). Here, we reported a new method for TORCH screening with five infections based on a concise and efficient bead-based microfluidic system. Five functionalized polycarbonate single beads, which respectively capture five IgGs from different pathogens, are fabricated to perform the sandwiched chemiluminescence immunoassay with the developed microfluidic chip for TORCH screening. To reduce the complexity of flow control as well as non-specific binding, multiple reaction chambers are incorporated to allow each reaction step to be separately completed in an independent chamber within the developed microfluidic chip. Each reaction chamber is divided into five interconnected independent cells respectively for five single beads. Each single bead with a ferrous core can be flexibly actuated by a controllable magnetic field for active mixing or convenient transporting. Qualitative detection for TORCH screening with five infections is achieved by analyzing the chemiluminescence signal collected with a CCD camera. Comparing to existing methods, rapid, automatic, convenient and low-cost TORCH screening with an integrated and disposable microfluidic chip can be achieved for point-ofcare diagnostics.

\section{Materials and methods}

\subsection{Single bead for TORCH screening}

To achieve TORCH screening with desired detection sensitivity, five single beads with a high surface-to-volume ratio are fabricated to respectively capture TOX-IgG, RUB-IgG, CMV-IgG, HSV-1-IgG and HSV-2-IgG. Each single bead ( $2 \mathrm{~mm}$ diameter) made of polycarbonate is able to detect one specific IgG corresponding to one infection through its functionalized surface. Similar to magnetic beads, two iron balls with a diameter of $0.6 \mathrm{~mm}$ are embedded in the single bead as the ferrous core, which allow the single bead to be conveniently actuated with a controllable magnetic field. As shown in Fig. 1, similarly, totally five single beads are fabricated for joint detection in TORCH screening.

Each single bead (as shown in Fig. 1) is first drilled by a $0.6 \mathrm{~mm}$ drilling bit with the depth of $1.5 \mathrm{~mm}$, and then two iron balls are pushed into the blind hole before it is sealed with hot melting. After that, the surface of each single bead is immobilized with one type of antigen for one type of IgG capturing. Non-specific immobilization based on physical absorption is adopted to functionalize each single bead. As shown in Fig. 1, joint qualitative detection for five infections in TORCH screening can be simultaneously performed with five functionalized single beads based on a sandwiched chemiluminescence immunoassay. First, IgG of each infection is specifically captured by one type of single bead through the immobilized antigen. And then, horseradish peroxidase (HRP) enzyme which is specifically captured through the second anti-human-IgG antibody triggers the intensive chemiluminescence for qualitative detection.

\subsection{Microfluidic chip for joint detection in TORCH screening}

A concise microfluidic chip, which consists of multiple reaction chambers, is developed to perform joint detection 
Fig. 1 The illustration of the chemiluminescence analysis with five independent single beads for TORCH screening. Each single bead is immobilized with a specific antigen first, and then the corresponding $\mathrm{IgG}$ of one infection is specifically captured by the single bead through the immobilized antigen. Finally, HRP enzyme is specifically captured by the single bead through the second anti-human-IgG antibody before it speeds up the

chemiluminescence with the mixed substrate buffers. The middle inset is a real picture of the single bead

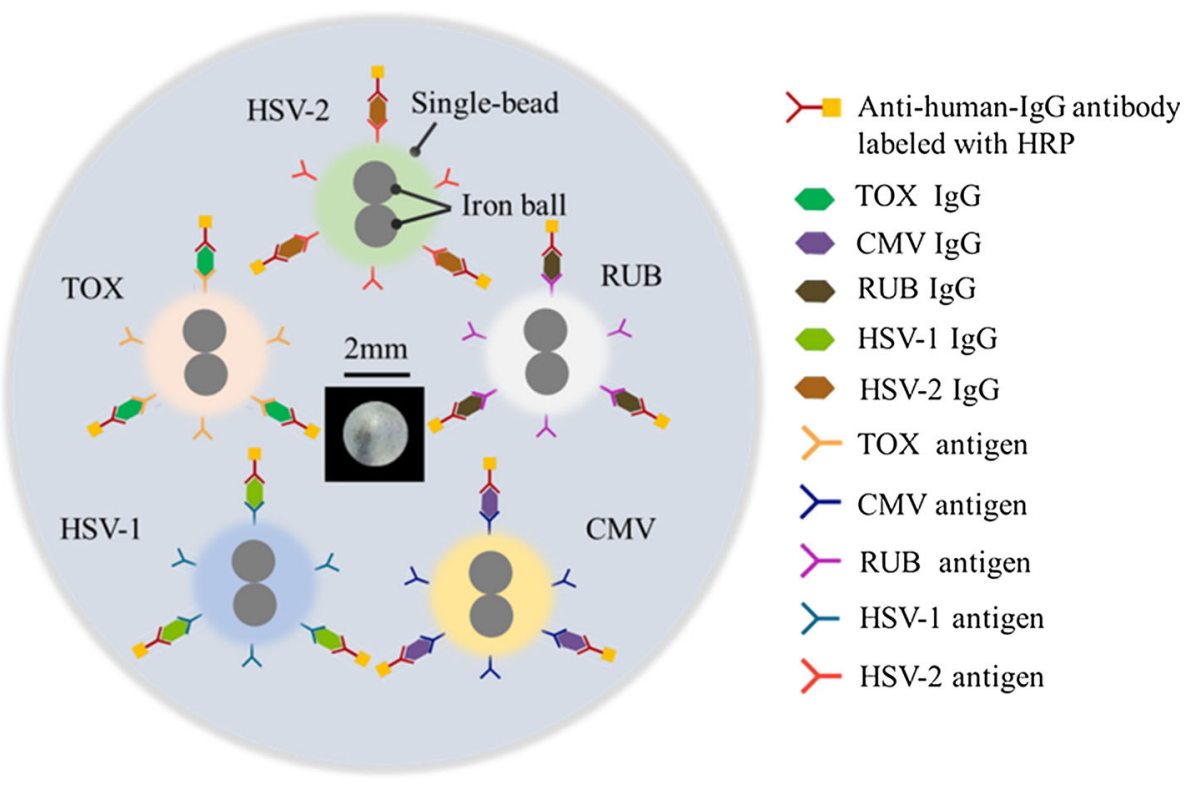

with five infections in TORCH screening by cooperating with five functionalized single beads. As shown in Fig. 2, the microfluidic chip $(270 \times 27 \times 14 \mathrm{~mm})$, which includes a reaction chamber $(11 \times 24 \times 4 \mathrm{~mm})$ for specific IgG capturing, four wash chambers $(11 \times 24 \times$ $4 \mathrm{~mm})$, a reaction chamber $(11 \times 24 \times 4 \mathrm{~mm})$ for HRP labeling, another four wash chambers $(11 \times 24 \times 4 \mathrm{~mm})$ and a chemiluminescence reaction chamber $(10 \times 24 \times$ $4 \mathrm{~mm}$ ), are fabricated by PMMA with acetonitrile based solvent bonding after laser ablation. As shown in Fig. 2, each reaction chamber is separated into five cells which are interconnected through holes $(1.5 \mathrm{~mm}$ in diameter) on the interfacing wall between each two adjacent cells, and five single beads can be respectively loaded into five different cells for simultaneous detection of five infections with one test sample. As shown in Fig. 2, a magnet is applied to actuate each single bead with a controllable magnetic field. With magnetic control, when each single bead is incubated with reagent in one cell, it can be moved back and forth to introduce active mixing to improve reaction efficiency, and meanwhile, it can be conveniently transported between each two adjacent reaction chambers by following a guiding path (a slope with a $35^{\circ}$ angle). Once the serum sample is manually loaded into the reaction chamber with five single beads in different cells, TORCH screening with five infections can be performed by sequentially transporting each single bead between each two adjacent reaction chambers with preloaded reagents. With the configuration of multiple reaction chambers, there has no need for any waste storage chamber since the waste reagent
Fig. 2 Microfluidic chip with multiple single beads for TORCH screening with five infections

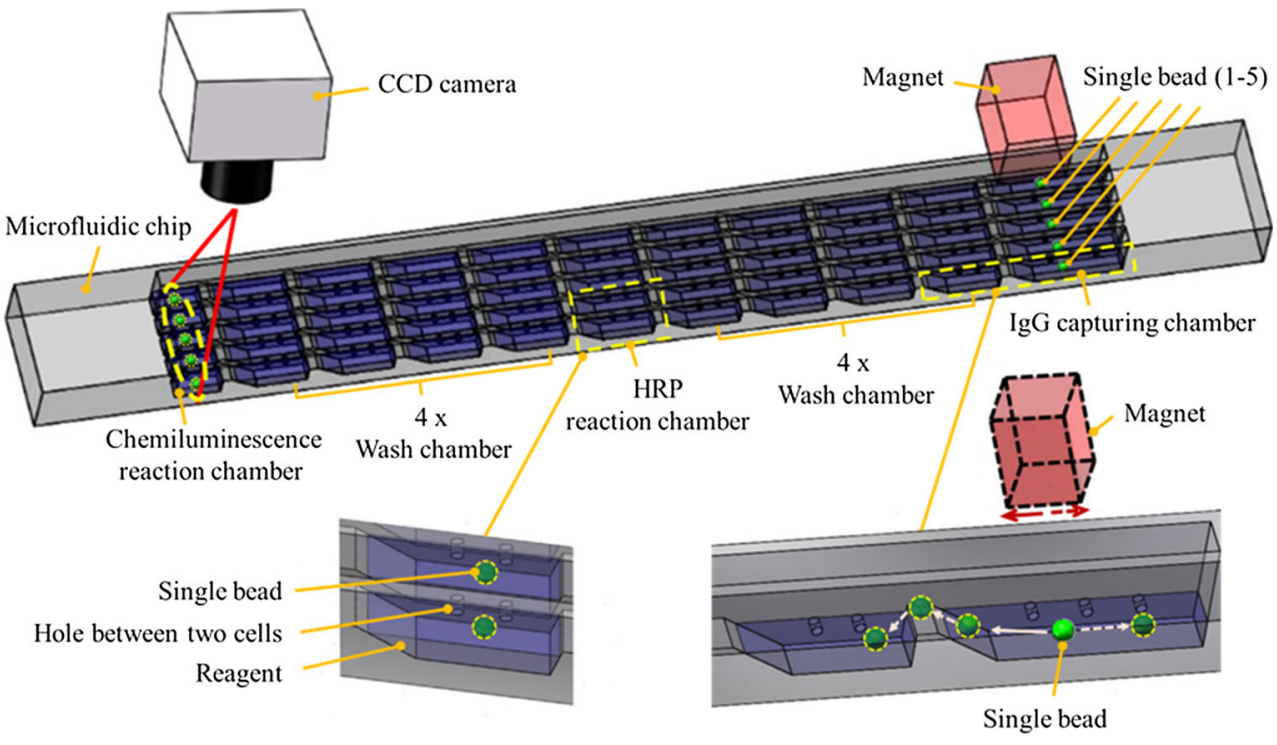


is always left behind after each single bead is transported to the next adjacent chamber.

As shown in Fig. 2, a permanent magnet, which is mounted on a linear moving stage, is used to actuate each single bead for enhanced mixing or convenient transporting. As shown in Fig. 2, assisted by the magnetic force, each single bead can be pulled toward the next adjacent reaction chamber along the guiding path. With multiple reaction chambers, each reaction can be performed in an independent chamber when each single bead is continually transported between each two adjacent chambers, which is beneficial to reduce non-specific reaction caused by nonspecific binding. With a concise microfluidic chip, efficient TORCH screening with five single beads can be completed without complicated flow control, which is helpful to achieve convenient, simple and low-cost detection at POC testing.

\subsection{Instrument for TORCH screening with microfluidic chip}

As shown in Fig. 3, a portable instrument $(340 \times 280 \times 175 \mathrm{~mm})$, which consists of a linear actuation module, a heating module and a CCD module, is developed to perform TORCH screening with the beadbased microfluidic chip. The linear actuation module, which includes a stepmotor, a belt, a positioning strip with multiple thin slots and a tray, is used to move the mounted magnet to achieve a controllable magnetic field for different purposes, for example, to move each single bead back and forth for enhanced mixing, or to transport each single bead between each two adjacent reaction chambers. With the positioning strip, the movement of the magnet can be effectively controlled by detecting its position through multiple thin slots on the positioning strip. As shown in Fig. 3, only one magnet is required to implement TORCH screening by applying synchronous magnetic control to five single beads within a streamlined microfluidic chip.

As shown in Fig. 3, a 16-bit charge coupled device (CCD) camera (ST-1603, SBIG) is used to collect detection signal by taking the chemiluminescence picture from five single beads. The CCD camera is controlled by a laptop through custom software developed with Visual Studio 2010. The original chemiluminescence picture is analyzed by a custom image processing algorithm to achieve the signal intensity for each single bead. A resistance heater (not shown in Fig. 3) is applied to heat the microfluidic chip at $37{ }^{\circ} \mathrm{C}$ from the bottom to further improve the reaction efficiency. Except the CCD camera, all the electrical and mechanical modules as wells as the microfluidic chip are enclosed in a small box to avoid negative effect from the outside environmental light.

\subsection{Chemiluminescence analysis for TORCH screening with microfluidic chip}

First, $700 \mu \mathrm{L}$ serum sample is manually loaded into one of the reaction chambers with five single beads and incubated for around $10 \mathrm{~min}$ for $\mathrm{IgG}$ capturing. Meanwhile, as do in the next following steps, all the single beads are moved back and forth within the reaction chamber with a speed of $1.5 \mathrm{~mm} / \mathrm{s}$ through magnetic control. After that, all the single beads are transported into the next wash chamber with preloaded $700 \mu \mathrm{L}$ wash buffer and incubated for $1 \mathrm{~min}$, and totally four time wash are respectively competed in different chambers. Second, all the single beads are transported into the next reaction chamber with preloaded $700 \mu \mathrm{L}$ HRP and incubated for $10 \mathrm{~min}$ for specific enzyme binding. After that, all the single beads are transported into the next wash chamber with preloaded $700 \mu \mathrm{L}$ wash buffer and incubated for $1 \mathrm{~min}$, and totally four time wash are respectively competed in different chambers. Finally, all the single beads are transported into the
Fig. 3 Instrument for TORCH screening with microfluidic chip

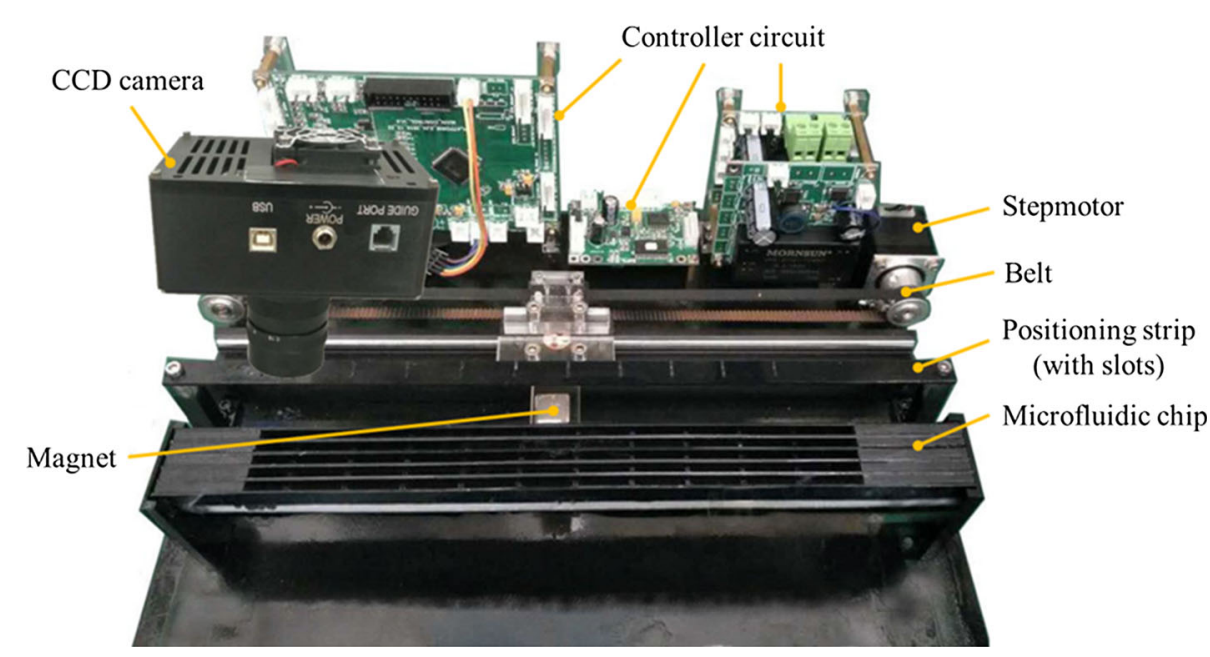


reaction chamber with preloaded $500 \mu \mathrm{L}$ of two mixed substrate buffers with $250 \mu \mathrm{L}$ for each of them for chemiluminescence detection. Qualitative detection is achieved by comparing the intensity of the chemiluminescence signal of each single bead with a predefined cutoff value. The detection sensitivity of TORCH screening can be improved by the active mixing introduced by the movement of the single bead based on magnetic control. With the single-bead based microfluidic chip, TORCH screening can be completed within $30 \mathrm{~min}$ with significantly reduced non-specific binding.

\section{Results and discussion}

It has been found that when the microfluidic chip is made from transparent PMMA, there will have remarkable reflection of chemiluminescence signal from the inside wall of the reaction chamber around each single bead, which is undesired for accurate analysis to the detection signal. In contrast, when black PMMA is applied, low and clear background around each single bead can be achieved when a CCD camera is used to collect chemiluminescence signal from the reaction chamber. Therefore, black PMMA is adopted for chip fabrication. To achieve reliable and stable detection, the mean value of the chemiluminescence signal intensity readings from the CCD camera (exposure time $1 \mathrm{~min}$ ) in the first two min are adopted as the detection result. A custom image processing algorithm was adopted to achieve the chemiluminescence signal intensity for the single bead. Serum samples were used in all experiments. Qualitative test is performed for TORCH screening, and for each infection, a proper cutoff value is predefined to determine the detection result (positive or negative) based on the detected signal amplitude from one single bead.

\subsection{Chemiluminescence analysis with different reaction temperatures}

To find out the optimal operation temperature for chemiluminescence analysis with microfluidic chip, two operation modes with different working temperatures were compared between each other. For the mode I, the sandwiched chemiluminescence analysis is performed with the developed microfluidic chip at room temperature, for example, around $25^{\circ} \mathrm{C}$, while for the mode II, the microfluidic chip is heated to $37{ }^{\circ} \mathrm{C}$ from the bottom by a resistance heater during reaction. Except the difference on the reaction temperature, all the other settings with the experiment are the same in both modes. In the experiment, serum sample of CMV positive was chosen. As shown in Fig. 4, when the microfluidic chip is heated up to $37{ }^{\circ} \mathrm{C}$,

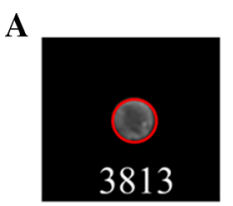

Without heating

$\left(25^{\circ} \mathrm{C}\right)$
B

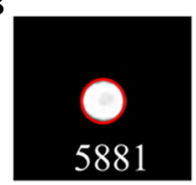

With heating

$\left(37{ }^{\circ} \mathrm{C}\right.$ )
Fig. 4 Chemiluminescence image of the single bead (marked with a red circle) in the reaction chamber, which is achieved without (a) and with (b) heating

the intensity of chemiluminescence signal subtracted by the background from the single bead is significantly increased compared with that achieved at room temperature. The experiment was repeated for three times, and similar results were attained.

As shown in Fig. 4, the signal amplitude can be increased up to 1.54 times (from 3813 to 5881) when the reaction efficiency is improved with an optimal working temperature at $37^{\circ} \mathrm{C}$, which is helpful to improve the detection sensitivity with high signal readings. Therefore, to achieve sensitive bead-based TORCH screening with the sandwiched chemiluminescence analysis, the microfluidic chip was always heated at $37^{\circ} \mathrm{C}$ in the following experiments.

\subsection{Chemiluminescence analysis with single-bead introduced active mixing}

To find out the optimal operation mode for chemiluminescence analysis with microfluidic chip, two operation modes with different mixing methods were compared between each other. For the mode I, the sandwiched chemiluminescence analysis is performed with the developed microfluidic chip where mixing is achieved based on natural diffusion when the single bead keeps static, while for the mode II, active mixing is introduced by continually moving the single bead back and forth with a magnet. Except the difference on the mixing mode, all the other settings with the experiment are the same to both modes. In the experiment, serum sample of RUB positive was chosen. As shown in Fig. 5, when active mixing is introduced by

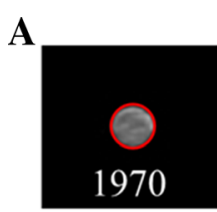

Diffusion

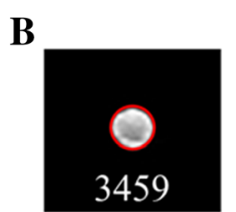

Active mixing
Fig. 5 Chemiluminescence image of the single bead (marked with a red circle) in the reaction chamber, which is achieved with diffusion (a) and active mixing (b) 
the single bead, the intensity of chemiluminescence signal subtracted by the background from the single bead is significantly increased compared with that achieved with the static single bead. The experiment was repeated for three times, and similar results were attained.

As shown in Fig. 5, the signal amplitude can be increased up to 1.76 times (from 1970 to 3459) when the reaction efficiency is improved based on active mixing. When the single bead is moved back and forth inside the reaction chamber, it will take short time for the detection signal of the single bead to reach a reasonably high reading, which is helpful to decrease the required detection time for TORCH screening. Therefore, to achieve sensitive TORCH screening, active mixing was introduced by the movement of the single bead based on magnetic control in the following experiments.

\subsection{Sensitivity evaluation of TORCH screening with microfluidic chip}

The sensitivity of the developed method for TORCH screening with microfluidic chip was evaluated and compared with ELISA. Five types of positive serum samples respectively related with five different pathogens in TORCH screening were used in the experiment. Since qualitative detection is adopted by TORCH screening with microfluidic chip, instead of using a limit of detection as evaluation parameter, the sensitivity of the developed method is evaluated based on its comparison with ELISA.

First, each type of positive serum sample with the original concentration was detected, and then it was serially diluted with two folds for each time for further experiment. Meanwhile, parallel experiments were performed with ELISA. For ELISA, OD (optical density) value for each test was read by a typical plate reader. Experiment with each type of positive serum sample was repeated for at least three times, and similar results were achieved. As an example, the experimental results with a serum sample of TOX positive for both the developed microfluidic system and ELISA are shown in Fig. 6.

Figure $6 \mathrm{a}, \mathrm{b}$ are respectively the experimental results with the microfluidic system and ELISA, and Fig. $6 \mathrm{c}$ includes the normalized results with both methods. As shown in Fig. 6, the developed microfluidic system and ELISA have comparable sensitivity since both of them work well until the original serum sample is diluted down to 32 folds. When the original sample is further diluted, it is difficult for both of them to differentiate between 32-fold dilution and 64-fold dilution. Similar experimental results were attained for other four infections (data not shown here), for example, RUB, CMV, HSV-1 and HSV-2. Comparing to ELISA, rapid and automated TORCH screening with acceptable sensitivity can be achieved with an easy-to-operate and low-cost microfluidic system, which is beneficial to POC testing at resource-poor settings.
Fig. 6 Experimental results with serum samples of TOX positive, which are receptively achieved with the developed microfluidic system (a) and ELISA (b). c The normalized results with both methods
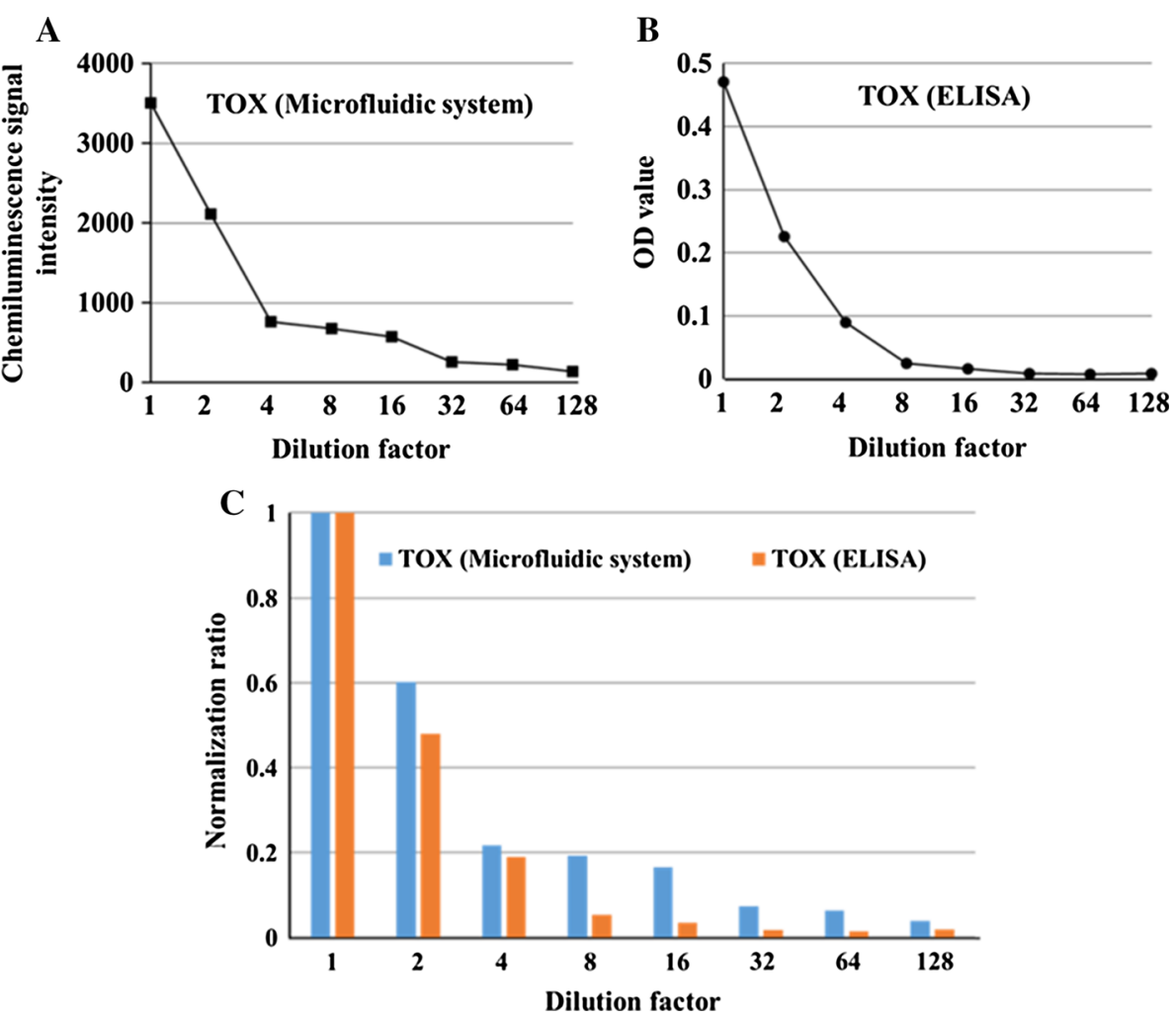


\subsection{Decision of cutoff value for TORCH screening}

To find out proper cutoff values for qualitative detection in TORCH screening, multiple test samples including typical low positive and negative samples were detected with the developed microfluidic system. Especially, negative samples with high signal reading were chosen to resolve more reasonable cutoff values for robust qualitative analysis. Before experiment, all serum samples were detected and verified with ELISA. For each infection, three low positive samples as well as three negative samples were detected to achieve a proper cutoff value based on their chemiluminescence signal intensity. As shown in Fig. 7, there is significant difference of signal readings between low positive and negative samples for each infection.

As shown in Fig. 7, for TOX, the difference of signal readings between low positive and negative samples is smaller than those of other infections, for example, CMV, RUB, HSV-1 or HSV-2. For each infection, the average value of the readings from both the low positive and the negative samples is chosen as the cutoff value, for example, TOX: 1117.5, CMV: 2203, RUB: 667.5, HSV-1: 2570, HSV-2: 3580.5. In TORCH screening, the cutoff value for each infection can be used in qualitative detection to determine the test result (positive or negative).

\subsection{Evaluation of the developed microfluidic system with serum samples}

The performance of the developed microfluidic system for TORCH screening was evaluated with totally six serum samples (provided by Beijing Wantai Biological Pharmacy Enterprise Co., Ltd.). Parallel experiments were performed with ELISA for verification. The detection results of TORCH screening with the developed microfluidic system are shown in Fig. 8.

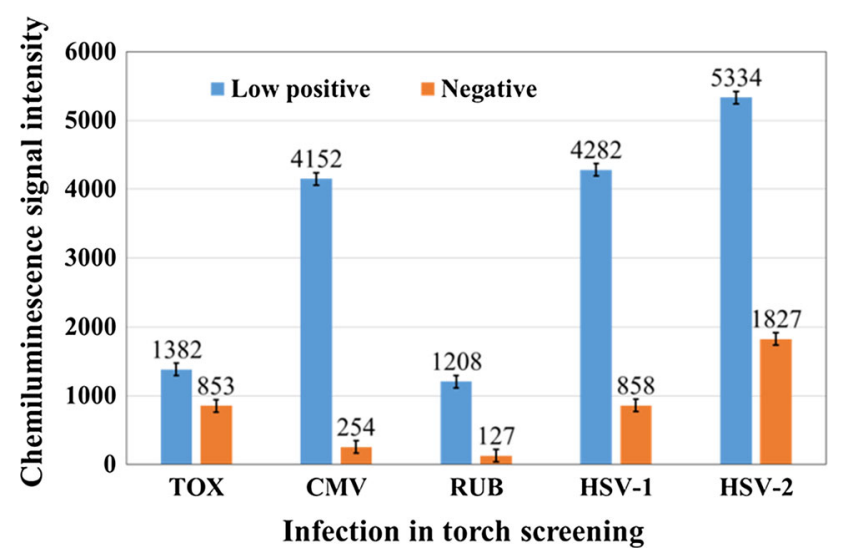

Fig. 7 Chemiluminescence signal intensity with typical low positive and negative samples for each infection in TORCH screening
The original chemiluminescence signal for each single bead is shown in Fig. 8a, and the corresponding chemiluminescence signal intensity for each single bead is shown in Fig. 8b. As shown in Fig. 8, finally, qualitative analysis for six serum samples can be performed by comparing the chemiluminescence signal intensity with the corresponding cutoff value for each infection. For sample A, TOX, CMV and HSV-2 are negative, RUB and HSV-1 are positive. For sample B, TOX, CMV and HSV-2 are negative, RUB and HSV-1 are positive. For sample C, TOX, and HSV-2 are negative, CMV, RUB and HSV-1 are positive. For sample D, TOX, and HSV-2 are negative, CMV, RUB and HSV-1 are positive. For sample E, TOX, and HSV-2 are negative, CMV, RUB and HSV-1 are positive. For sample F, TOX, HSV- 1 and HSV-2 are negative, CMV and RUB are positive. The experiment was repeated for at least three times and consistent results were achieved. The qualitative detection results with the developed microfluidic system agree with those achieved with traditional ELISA, which confirm its accuracy for TORCH screening.

As a proof of concept, it has been demonstrated that simple, convenient and efficient TORCH screening can be easily performed with the developed microfluidic system based on multiple functionalized single beads within $30 \mathrm{~min}$. Five infections can be automatically detected and analyzed in parallel with one test sample, which is especially helpful to reduce detection time and cost. Comparing to other existing methods, such as ELISA (at least $1 \mathrm{~h}$ detection time), it is more desirable for the developed method to be adopted in point-of-care diagnosis at resource poor settings.

\section{Conclusions and outlook}

A microfluidic system based on multiple single beads has been developed to perform TORCH screening at POC testing. Five polycarbonate single beads, which are respectively immobilized with different antigens for TORCH screening, are fabricated to perform parallel detection to five different infections. Each single bead of $2 \mathrm{~mm}$ in diameter is embedded with a ferrous core to allow it to be conveniently actuated by an outside magnet. The disposable microfluidic chip, which consists of multiple reaction chambers respectively for different purposes, is developed to implement TORCH screening based on the sandwiched chemiluminescence immunoassay. With magnetic actuation, each single bead can be conveniently transported between each two adjacent reaction chambers with preloaded reaction buffers for the next reaction once the last one is completed, which is helpful to reduce nonspecific reaction caused by non-specific binding. On the other hand, with another mode of magnetic actuation, the 
Fig. 8 Detection result of TORCH screening with the developed microfluidic system. a Original chemiluminescence signal of each single bead (marked with a red circle) in TORCH screening with five infections for six test samples (A-F). b Analyzed chemiluminescence signal intensity of each single bead in TORCH screening
A

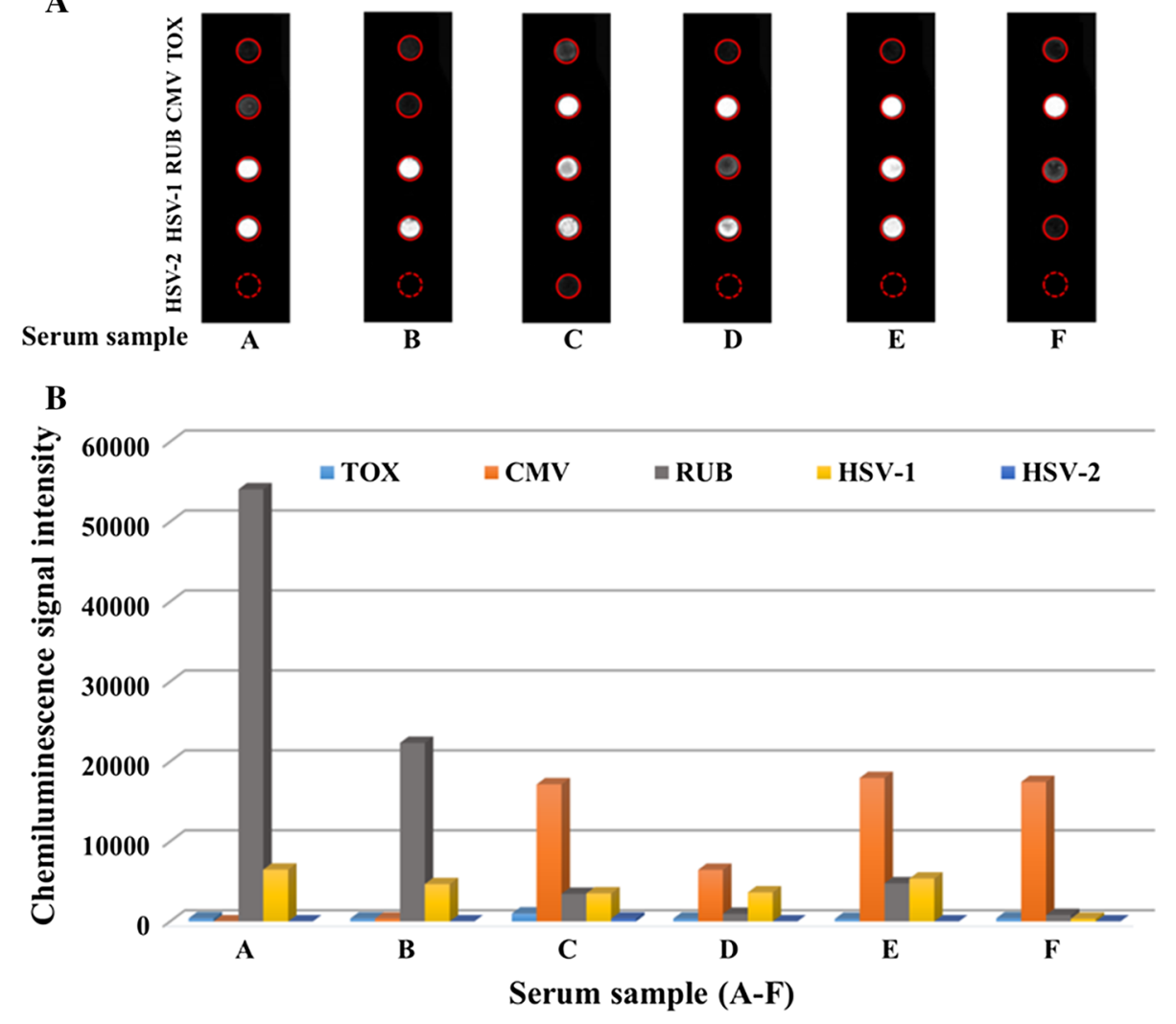

single bead can be moved back and forth within the reaction chamber to improve the detection sensitivity with active mixing.

With a configuration for high throughput detection, the developed microfluidic system is able to perform TORCH screening by introducing synchronous magnetic control to five single beads with a common magnet. A sensitive CCD camera is adopted to simultaneously collect chemiluminescence signal from five single beads by taking picture with a preset exposure time, and the original signal is further analyzed with a custom image processing algorithm to achieve the chemiluminescence signal intensity for each single bead. Qualitative detection is performed by comparing the chemiluminescence signal intensity of each single bead with the corresponding cutoff value for each infection. When the microfluidic chip is heated to around $37{ }^{\circ} \mathrm{C}$, the chemiluminescence signal intensity of the single bead will be significantly increased because of the improved reaction efficiency comparing to that achieved at room temperature. Comparing to ELISA, comparable sensitivity is achieved with the developed microfluidic system for accurate and reliable TORCH screening. The cutoff value for each infection in TORCH screening is properly decided based on the chemiluminescence signal readings from both low positive and negative samples. The performance of the developed microfluidic system is further evaluated with serum samples, and accurate and efficient TORCH screening can be completed within just 30 min, which is beneficial to POC diagnosis.

In summary, a simple, convenient, easy-to-operate and low-cost microfluidic system has been properly developed to perform TORCH screening with multiple functionalized single beads. A concise instrument, which consists of a linear actuation module, a heating module and a CCD camera, is adopted to facilitate chemiluminescence reaction and detection with the disposable microfluidic chip. Comparing to other existing methods, with the developed microfluidic system, TORCH screening can be automatically performed with reduced cost and time. In principle, with adjustable functionalized single beads, the developed microfluidic system can be flexibly programmed for diagnosis of one or multiple infections related with TORCH. Meanwhile, in terms of a high throughput analysis platform, the developed microfluidic system can be potentially modified to accommodate other joint disease diagnosis when specifically functionalized single beads are available for the detection of different biomarkers.

Acknowledgements The work was supported by foundation to Beijing Engineering Research Center of Diagnostics in Infectious Diseases (Beijing Wantai Biological Pharmacy Enterprise Co., Ltd.) for incubation and development of scientific and technological innovation base from Beijing Municipal Commission of Science and 
Technology (No. Z16110005016094), the National Natural Science Foundation of China (No. 81371711) and the research fund to the top scientific and technological innovation team from Beijing University of Chemical Technology (No. buctylkjcx06).

\section{Compliance with ethical standards}

Conflict of interest The authors declare that they have no conflict of interest.

\section{References}

Barbi M, Binda S, Caroppo S, Primache V (2006) Neonatal screening for congenital cytomegalovirus infection and hearing loss. J Clin Virol 35:206-209

Chen D, Mauk M, Qiu X, Liu C, Kim J, Ramprasad S, Ongagna S, Abrams WR, Malamud D, Corstjens PL, Bau HH (2010) An integrated, self-contained microfluidic cassette for isolation, amplification, and detection of nucleic acids. Biomed Microdevices 12:705-719

Choi G, Song D, Shrestha S, Miao J, Cui L, Guan W (2016) A fielddeployable mobile molecular diagnostic system for malaria at the point of need. Lab Chip 16:4341-4349

Chung CY, Wang JC, Chuang HS (2016) Rapid bead-based antimicrobial susceptibility testing by optical diffusometry. PLoS One 11:e0148864

Du K, Cai H, Park M, Wall TA, Stott MA, Alfson KJ, Griffiths A, Carrion R, Patterson JL, Hawkins AR, Schmidt H, Mathies RA (2017) Multiplexed efficient on-chip sample preparation and sensitive amplification-free detection of Ebola virus. Biosens Bioelectron 91:489-496

Gong MM, Sinton D (2017) Turning the page: advancing paper-based microfluidics for broad diagnostic application. Chem Rev 117:8447-8480

Jiang L, Yu Z, Du W, Tang Z, Jiang T, Zhang C, Lu Z (2008) Development of a fluorescent and colorimetric detection methods-based protein microarray for serodiagnosis of TORCH infections. Biosens Bioelectron 24:376-382

Jong EPD, Vossen ACTM, Walther FJ, Lopriore E (2013) How to use... neonatal TORCH testing. Arch Dis Child Educpract 98:93

Li D, Yang H, Zhang WH, Pan H, Wen DQ, Han FC, Guo HF, Wang XM, Yan XJ (2006) A simple parallel analytical method of prenatal screening. Gynecol Obstet Invest 62:220-225

Li X, Niu Y, Chen Y, Wu D, Yi L, Qiu X (2016) Microfluidic paperbased sample concentration using ion concentration polarization with smartphone detection. Micromachines 7:1

Lim CT, Zhang Y (2007) Bead-based microfluidic immunoassays: the next generation. Biosens Bioelectron 22:1197-1204

Liu C, Qiu X, Ongagna S, Chen D, Chen Z, Abrams WR, Malamud D, Corstjens PLAM, Bau HH (2009) A timer-actuated immunoassay cassette for detecting molecular markers in oral fluids. Lab Chip 9:768-776

López-Marzo AM, Merkoçi A (2016) Paper-based sensors and assays: a success of the engineering design and the convergence of knowledge areas. Lab Chip 16:3150-3176

Mosley O, Melling L, Tarn MD, Kemp C, Esfahani MM, Pamme N, Shaw KJ (2016) Sample introduction interface for on-chip nucleic acid-based analysis of Helicobacter pylori from stool samples. Lab Chip 16:2108-2115

Neu N, Duchon J, Zachariah P (2015) TORCH infections. Clin Perinatol 42:77-103

Qiu X, Thompson JA, Chen Z, Liu C, Chen D, Ramprasad S, Mauk MG, Ongagna S, Barber C, Abrams WR, Malamud D, Corstjens PLAM, Bau HH (2009) Finger-actuated, self-contained immunoassay cassettes. Biomed Microdevices 11:1175-1186

Qiu X, Liu C, Mauk MG, Hart RW, Chen D, Qiu J, Kientz T, Fiene J, Bau HH (2011a) A portable analyzer for pouch-actuated, immunoassay cassettes. Sens Actuators B 160:1529-1535

Qiu X, Chen D, Liu C, Mauk MG, Kientz T, Bau HH (2011b) A portable, integrated analyzer for microfluidic-based molecular analysis. Biomed Microdevices 13:809-817

Revello MG, Vauloup-Fellous C, Grangeot-Keros L, Helden JV, Dickstein Y, Lipkin I, Mühlbacher A, Lazzarotto T (2012) Clinical evaluation of new automated cytomegalovirus IgM and IgG assays for the Elecsys ${ }^{\circledR}$ analyser platform. Eur J Clin Microbiol Infect Dis 31:3331-3339

Stumpf F, Schwemmer F, Hutzenlaub T, Baumann D, Strohmeier O, Dingemanns G, Simons G, Sager C, Plobner L, von Stetten F, Zengerle R, Mark D (2015) LabDisk with complete reagent prestorage for sample-to-answer nucleic acid based detection of respiratory pathogens verified with influenza A H3N2 virus. Lab Chip 16:199-207

Sun J, Xianyu Y, Jiang X (2014) Point-of-care biochemical assays using gold nanoparticle-implemented microfluidics. Chem Soc Rev 43:6239-6253

Ursu RG, Costin D, Iancu LS, Nemescu D (2015) The clinical utility of TORCH testing of pregnant women from northeastern Romanian population: Application of electrochemiluminiscence assay. In: IEEE International Conference on E-Heatlh and Bioengineering. Iaúi, Romania

Van HJ, Grangeotkeros L, Vauloupfellous C, Vleminckx R, Masset F, Revello MG (2014) Evaluation of fully automated assays for the detection of Rubella IgM and IgG antibodies by the Elecsys $\left({ }^{\circledR}\right)$ immunoassay system. J Virol Methods 199:108-115

Wu D, Zhang J, Xu F, Wen X, Li P, Zhang X, Qiao S, Ge S, Xia N, Qian S, Qiu X (2017) A paper-based microfluidic Dot-ELISA system with smartphone for the detection of influenza A. Microfluid Nanofluid 21:43

Yang H, Guo Q, He R, Li D, Zhang X, Bao C, Hu H, Cui D (2009) A quick and parallel analytical method based on quantum dots labeling for ToRCH-related antibodies. Nanoscale Res Lett 4:1469-1474

Yang K, Peretz-Soroka H, Liu Y, Lin F (2016) Novel developments of mobile sensing based on the integration of microfluidic devices and smartphone. Lab Chip 16:943-958

Zhang D, Liu Q (2016) Biosensors and bioelectronics on smartphone for portable biochemical detection. Biosens Bioelectron $75: 273-284$

Zhang H, Liu L, Fu X, Zhu Z (2013) Microfluidic beads-based immunosensor for sensitive detection of cancer biomarker proteins using multienzyme-nanoparticle amplification and quantum dotslabels. Biosens Bioelectron 42:23-30

Zhang L, Ding B, Chen Q, Feng Q, Lin L, Sun J (2017) Point-of-caretesting of nucleic acids by microfluidics. TrAC Trends Anal Chem 94:106-116 